\title{
Nanoscale Mechanical Scattering Experiments towards the Local Analysis of Thermal Active Modes and Dispersion Interactions at Interfaces
}

\author{
René M. Overney \\ Department of Chemical Engineering, University of Washington, Benson Hall, Seattle, WA \\ 98195
}

Thermal active modes, i.e., molecular and submolecular mobilities, inherent to the relaxation behavior of solid condensed organic matter, such as polymers and molecular self-assemblies, and, interfacial and surface free energies, are of great importance for many practical applications. For instance, in organic second-order nonlinear optical materials (NLO), pursued for photonic device applications, the molecular mobility plays a pivotal role in producing stable NLO materials of high poling efficiency [1]. Other applications such as frictional sliding systems or nanocomposites, both involving nanoconstrained materials, depend on the surface/interfacial energies. Common to thermal modes and interfacial forces are that they are difficult to quantify, in particular, in systems that either possess an amorphous complex entropic structure, or are of small size.

From scanning force microscopy (SFM) an approach has been devised over the years, providing direct and local insight into thermal modes and interfacial forces via an energetic analysis that is based on the time-temperature superposition principle [2]. Dubbed "intrinsic friction analysis" (IFA), it utilizes the mechanical scattering process between a sliding SFM tip in contact with the thermal active modes of the scanned sample. In the past, in particular, rotational and translational modes have been investigated involving complex organic systems, such as polymers and organic molecular glasses [2-5]. Recently, also molecular binding interactions [1] and surface dispersion interactions [6] could be energetically analyzed with IFA. In this paper, we will focus on both aspects, namely the molecular mobility in self-assembled systems depending on the molecular interaction strength, and the quantum electro-dynamic binding fluctuations between Van der Waals interacting surfaces.

Based on a prior study involving dendritic organic non-linear optical (NLO) self-assembly molecular glasses, Fig. 1(a), an intermediate thermal phase regime was discovered that was found pivotal for both the phase stability and the degree of non-linear polarization. In the first part of this presentation, we focus on the energetic analysis of this intermediate phase regime in contrast to the two bordering "low" and "high" temperature phase regimes, Fig. 1(b), as function of the dendritic interactions, involving arene-perfluoroarene moieties, and the strength of the intrinsic molecular dipole fields. The presence of dendritic groups was found to fundamentally alter transition temperatures and the molecular relaxation behavior. Based on enthalpic and entropic energetic IFA analyses, thermally active modes in the low temperature regime were found to be intimately connected to the dendron structure, while in the intermediate phase regime a substantial amount of the total energy was found to be of cooperative entropic nature, Fig. 1(c). The multiple interactions (from dipole-dipole interactions to local non-covalent dendritic interactions) are discussed and summarized in a model that describes the thermal transitions and phases. 
While we focus in the first part in this presentation on the energetics in molecular mobility in self-assembled systems, in the second part, we will direct the discussion to IFA's applicability to spontaneous quantum electrodynamic fluctuations, i.e., Van der Waals interactions, between inorganic substances, Fig. 2, involving materials, such as layered materials (e.g., graphene, or molybdenum disulfide) and ionic materials (calcium fluoride). This study reveals IFA's ability to directly determine the Hamaker constant, and thus, gaining direct access locally to the interfacial energy, and consequently to the surface energy. Findings addressed here entail among others, good agreement of IFA data with the numerical analysis of EELS and optical reflectance data regarding Hamaker constant determination, and, a surprisingly strong reduction of the surface free energy from the macroscopic graphite system to single layer graphene.

\section{References}

[1] D. B. Knorr, S. J. Benight, B. Krajina, C. Zhang, L. R. Dalton, R.M. Overney, J. Phys. Chem. B, 116, (2012) 13793.

[2] D.B. Knorr, R.M. Overney, J. Chem. Phys., 129, (2008) 074504.

[3] S.E. Sills, T. Gray, R.M. Overney, J. Chem. Phys., 123, (2005) 134902.

[4] T. Gray et al., NanoLetters, 8, (2008) 754.

[5] D.B. Knorr, Jr., L. S. Kocherlakota, J. P. Killgore, and R. M. Overney, J. Membrane Sci., 346, ( 2010) 302.

[6] B.A. Krajina, L.S. Kocherlakota, R.M. Overney, J. Chem. Phys., 141, (2014) 164707
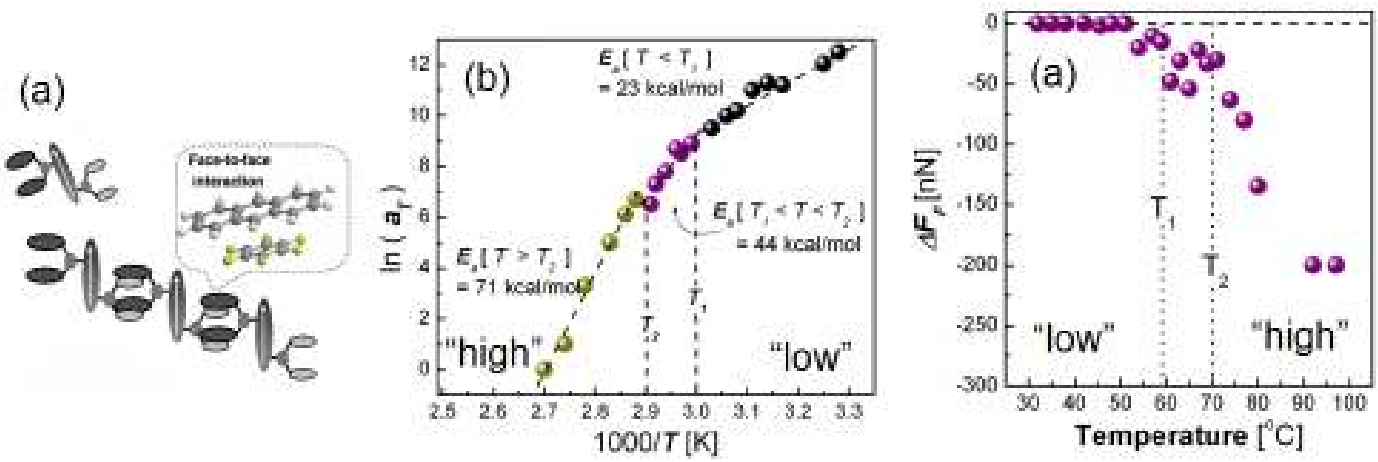

Figure 1. (a) Schematic self-assembly NLO material. (b) Energetics in low/intermediate and high temperature regime. (c) Cooperative entropy $\left(\Delta F_{F}\right)$ in the three temperature regimes.

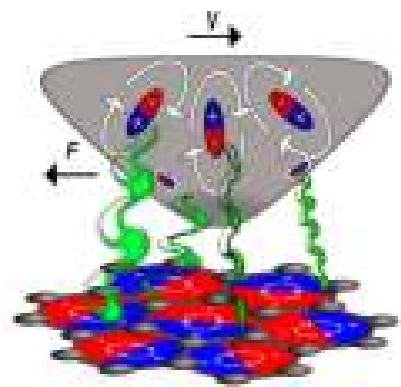

Figure 2. Spontaneous quantum electrodynamic fluctuations coupled to friction $F$ at velocity $v$ at a fixed temperature. 